stages is not unfavorable: in the advanced form, especially when the prostate on rectal examination gives the impression of being flabby and distended with secretion, a cure is more or less doubtful. Yet even in these cases we can afford a great deal of relief to our patient. Many a neurasthenic can be cured of his complaints, although a residuum of his prostatic affection remains. Careful and proper treatment can do much, even if it has to overcome many difficulties, and even if it leads to only partial success. But in the latter case also, the general condition of the patient will be materially improved. The treatment of the disease must not only be local but also constitutional. It should never be forgotten that most of our patients are neuropathic individuals, and we must therefore from the beginning endeavor to gain their full confidence. If local treatment relieves them of their principal annoyances we must impress upon them the curability of their disease, with the object to distract their minds from the hypochondriacal thoughts regarding their local affection. This general treatment will be essentially assisted by corroborative measures. For anemic or lymphatic individuals we provide tonics, fresh air, mild exercise. Carefully directed hydropathic treatment will often benefit materially. Our local therapeusis should, first of all, be prophylactic, that means we should do our very best to prevent the setting up of a prostatitis in all cases of gonorrheal urethritis. A careful treatment of the latter will in many cases enable us to avoid prostatic complications. If, however, acute prostatitis sets in, we should try to cure it as quickly and as completely as possible. An early detection of acute prostatitis before the inflammation has involved the whole gland, is the first step to a quick cure. I repeat, therefore, the necessity of examining every case of gonorrhea on this score. As soon as an acute prostatitis is diagnosed, we should put our patient to bed and stop all urethral injections and endo-urethral treatment. I am in the habit of prescribing the following treatment for my patients with acute prostatitis: Twice a day, morning and night, a rectal injection of warm water of at least 35 degrees $C$., care being taken that the nozzle of the irrigator does not hurt when introduced into the rectum. At least one pint of warm water should be injected and retained as long as possible. Before making injection at night a hip bath should be given, as warm as can comfortably be borne. After the rectal injection has returned, which usually is followed by a mild stool, a suppository of one-third of a grain of iodoform to one grain, is inserted in the rectum. Should pain be severe, and should micturition be frequent and painful, I add half a grain to a grain of codeia phosphoricum to the the suppository. Internally I give santal oil in capsules. By this simple treatment $I$ generally succeed in relieving pain almost instantaneously. This is usually followed, in the course of one or two weeks, by a subsidence of acute prostatitis, the prostate generally becoming almost normal to the touch. Of course, abscesses of the prostate, when already formed, have to be opened. It is, however, very rare that suppuration leading to an abscess takes place if my plan of treatment is instituted at an early stage. When, after the acute stage is overcome, chronic enlargement with all symptoms of chronic prostatitis remains, massage of the prostate is recommended.

Cases of an established chronic prostatitis, especially very old cases, are much more difficult to treat. In the great majority gentle massage of the prostate every second or third day will prove the most effective means of procuring, if not a complete cure, at least decided improvement. To hasten the reabsorption of the inflammatory exudations in the prostatic gland, I continue the warm rectal irrigations as prescribed for acute prostatitis, as well as the warm hip. bath and the iodoform suppositories. Lately I instituted another course of treatment besides massage of the prostate. An Arzberger rectal apparatus is introduced into the rectum and a stream of hot and cold water is conducted through it alternately. First a hot water stream runs three to five minutes through the double canula of the apparatus, and immediately after it a stream of cold water is passed through it. This is repeated until about 2 liters of each, hot and cold water, have made their circuit through the apparatus. By this alternate application of heat and cold in quick succession a rapid contraction and dilatation of the blood vessels of the prostate is produced, acting upon the gland almost like an electric current, only with less violence.

The first noticeable result of this treatment is that almost from very first application the patient experiences relief from the locul pains, which in the course of a few weeks generally disappear entirely. All my patients are very fond of this kind of treatment, and I can strongly recommend it. I repeat that massage of the prostate is regularly done at the same time.

All the suppositories except those of iodoform, prescribed for chronic prostatitis, have yielded so little good in my practice that I have given them up entirely. Of course it is understood, that in treating chronic prostatitis, the patient should be cured of his chronic gonorrheal urethritis at the same time.

\section{A NEW SIMPLEST PROCTOSCOPY.}

Presented to the Section of Surgery and Anatomy at the Forty-ninth Annual Meeting of the American Medical Association held at Denver, Colo., June 7-10, 1898.

BY THOS. CHAS. MARTIN, M.D.

LECTLRER ON DISFANES OF THE RECTLM IN THE CLEVELAND COLLEGE OF PHYSICIANS AND SURGEONS.

$$
\text { CLEVELAND, OHIO. }
$$

It has been complained that the best methods proposed for the inspection of the rectum require so expensive an armamentarium and such an infinite degree of painstaking practice on the part of him who would see, that the busy general practician can not hope to invade with his keen glance this darkest continent of special surgery. This is an error. On the contrary, as we shall see, no artificial means whatsoever are required for a complete ocular inspection of the rectum.

The elevation of the hips which sets in operation that principle of physics which governs the methods of Marion Sims' vaginal inspection (1845), Van Buren's rectal inspection (1871), and the method of the senior Allingham (1882), of Walter J. Otis (1887), and of Howard Kelly (1895), which controls my own procto-colonoscopy (1896), and which suggested Trendellenburg's posture, is, also, the chief feature of this the simplest proctoscopy.

The essentials of this method are a patient, an assistant and an operator having at least one finger on each hand. The patient is to be put into the kneechest posture ; the assistant is to put and to hold the patient; and the surgeon's fingers are to be used to open the anus, all in the following manner, to-wit:

1. The patient is to be completely anesthetized as 
he lies on his back, and then turned toward the assistant and into Sims' posture.

2. The assistant is to station himself at the patient's knees. In his left hand he is to grasp the patient's feet. $\mathrm{He}$ is to lean himself against the patient's knees. He is to pass his right arm under the patient's hips. Now steadying the foet and bearing himself firmly against the patient's knees, with his right arm he is to lift the hips and pull the patient into the knee-chest posture.

Here, securely in the embrace of the assistant, the patient is to be balanced on his perpendicular right thigh. Exactly here, throughout the whole time of the surgeon's manipulations, steadily the patient must be held.

3. The surgeon is to close his hand and to point his index finger as shown in the accompanying illustration. (Plate 1.) So, likewise the other hand. The wrists are to be crossed, the hands placed back against

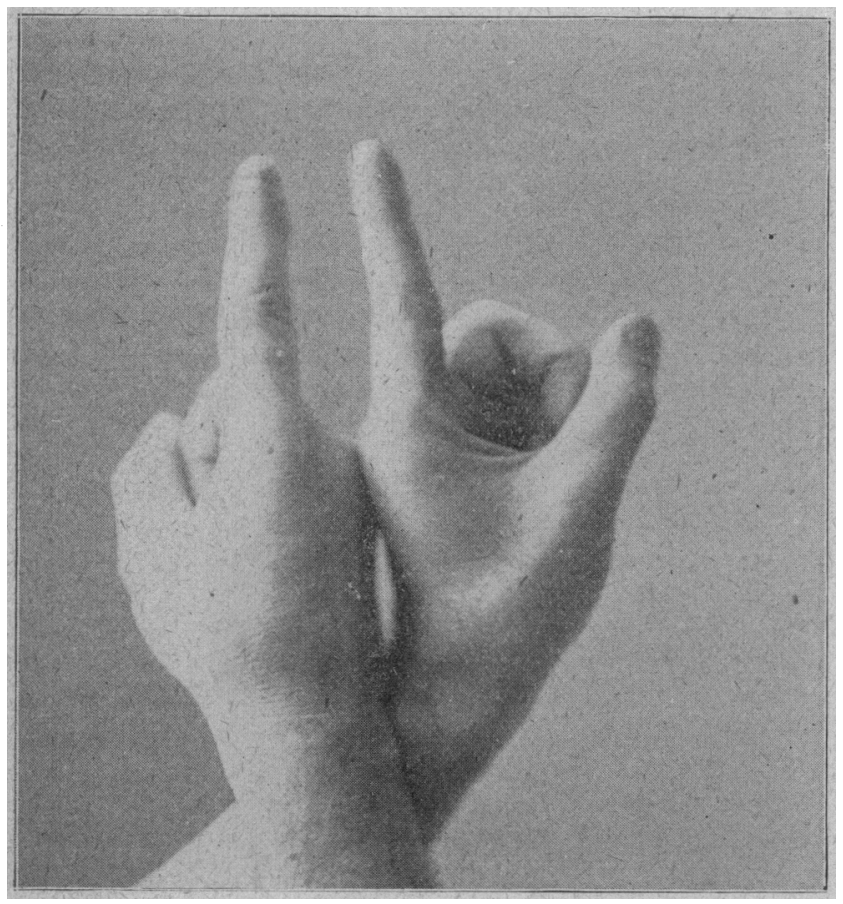

Frgure 1.

back, and the nails of the index fingers placed one against the other, as shown in the accompanying illustration. (Plate 2.) The surgeon is to lubricate these fingers and gently insinuate them through the anus and place their ends beyond the borders of the levatores ani. This accomplished, the anus is to be divulsed in the direction of the ischial tuberosities, by the surgeon forcibly parting his fingers as is shown in the accompanying illustration. (Plate 1.) Under this manipulation the rectum becomes atmospherically inflated.

Now, provided the surgeon lower his head to the level of his fingers and then rise again, or stoop, or move a little from side to side, he may command under his eye a view of the atmospherically inflated rectum to the depth of six or eight inches (15.24 or $20.32 \mathrm{~cm}$.) and in some instances he may behold even a part of the sigmoid flexure.

It is possible for the operator to manipulate his patient and to finish his inspection within two and a half or three minutes, provided the patient be in a state of complete anesthesia.
If this method be practiced, as I am persuaded it may be with facility by the general practitian, I am convinced that the greater number of rectal diseases may be instantaneously diagnosticated. But I must declare that here, at diagnosis, the achievement of the simplest proctoscopy ends, for the reason that the operator's hands are so full of his patient he can do nothing at all for the disease which he may have dis. covered.

Under some conditions and amid some circum. stances the rectum will not inflate. If there be a close stricture of the rectum, if there be malignant growth or other diseases of the rectum by means of which the gut's coats bave become extensively filled and fixed with an organized plastic exudate, if for some reason the intra-abdominal pressure be abnormally increased, as it may be by the voluntary bearing down of the patient, as it may be by enormous intestinal flatus, or by ascites, or if there be an inpinging

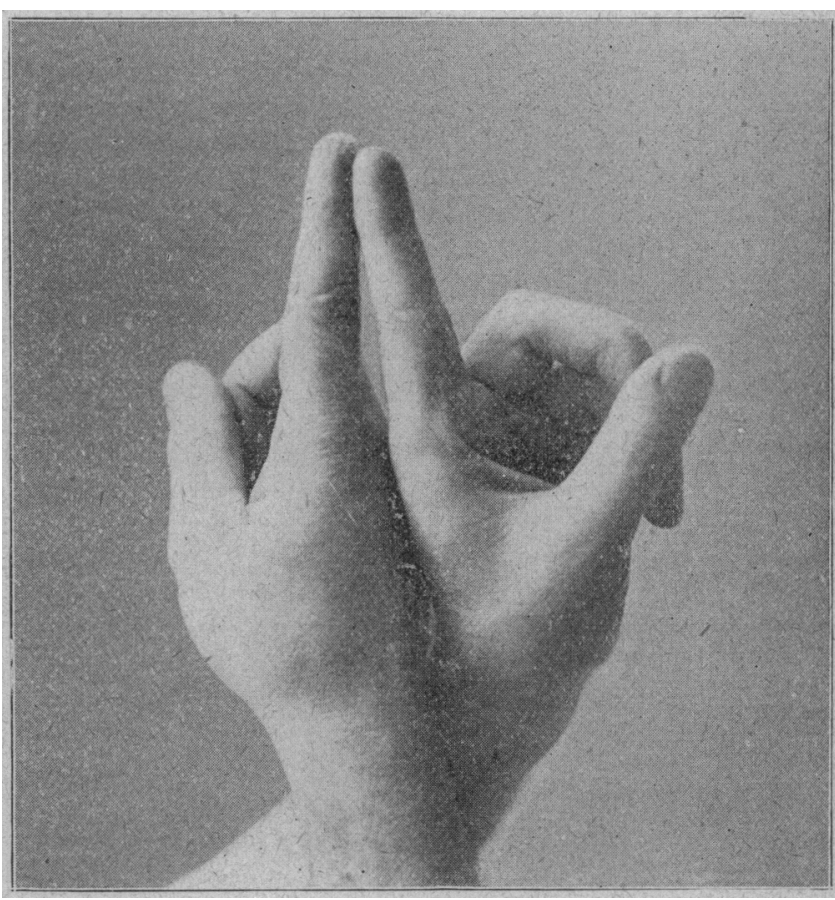

Figlie 2.

uterus, extra-rectal growth or extensive infiltrating disease of the contiguous textures, rectal inflation by this method or by any other which is governed by the same principle is a physical impossibility; but this need not baffle the man bent on seeing by instrumental aids.

Practiced as described, when not embarrassed by the exceptions specified, this method will achieve its purpose and reveal to the surgeon that the transverse diameter of the rectum is variable; while in some places it is not more than an inch $(3.54 \mathrm{~cm}$.$) , in others$ it is more than four times this diameter.

The rectum may present to the eye of the imaginative observer the appearance of a chain of urinary bladders, communicating one with another by means of irregularly elliptical openings set at varying axes, and bounded by the non-parallel borders of the semilunar valves. In the normal rectum the air pressure smooths the mucous membrane evenly over the entire surface of the gut. The normal mucous membrane of the so-called ampulla appears at first wet and of a 
shining bluish gray. As it dries, under the influence of gravitation the blue venous tint fades out of the gray and the wall becomes pink tinged. Presently it assumes the appearance of parchment, and sometimes it appears painted at rare intervals with ramifying little arteries which are crowded and overlapped by the larger companion veins; the latter are less arborescent and more suddenly dive and disappear in the bowel wall. In time, over all there comes a sheen as of collodion varnish and the vascular pictures fade save that one beneath the promonotory of the sacrum. These phenomena appear exactly as described only in the bealthy rectum; in the diseased organ the color varies much.

Should the operator deviate from the prescribed directions for the manipulation of his fingers and so twist his hands as to divulse the anus in the anteroposterior direction instead of laterally he invites de. feat upon himself; for in the male the fixation of the perineum and the immobility of the coccyx interfere with the requisite dilatation; while in the female, the extreme mobility of the perineum and particularly the backward displaceability of the coccyx will allow such traction to be made upon the levatores ani as to pull their inner borders parallel and almost together, and in consequence the wider the female's anus be opened anterio-posteriorly the closer it shuts laterally to rob us of our view.

1077 Prospect Street.

\section{PRIMARY CARCINOMA OF THE AXILLA.}

Presented to the Section on Surgery and Anatomy at the Forty-ninth Annual Meeting of the American Medical Association, held at Denver, Colo., June 7-10, 1898.

\section{BY D. W. GRAHAM, M.D.}

$$
\text { CHICAGO, ILL. }
$$

Carcinoma of the axilla consecutive to a primary focus of the disease in the mammary gland, or some other organ or structure, is a matter of almost daily occurrence in the experience of the surgeon. But primary carcinoma of the axillary region is rare enough to be worthy of discussion, especially as it raises some important questions in the fields of histologic anatomy and pathology, and of practice. There is no apparent reason why this region might not be the site of the squamous celled, or true skin carcinoma, as frequently as any other in the body. Primary cancer of the axillary skin is, however, exceedingly rare. Of 7297 primary cases of carcinoma analyzed by Williams 571 were of the skin, but of these latter only three were axillary.

There is no doubt but that occasionally a primary carcinoma of the breast will first manifest itself clinically through the infection and enlargement of the axillary lymphatics. The primary nodule, small, slowly-growing and deeply seated, and the conditions present for quick invasion and rapid progress of the lymphatic infection would give a pathologically secondary, but a clinically primary tumor in the axilla. While this is the explanation usually given and generally accepted for most carcinomatous tumors first appearing in this region it is applicable only to a small number of such cases, and should not apply except when the axillary tumor shows an exclusively lymphatic histologic basis.

There are two structures found in the axillary space in which carcinoma is prone to originate, which will account for nearly all cases of true primary carcinoma in this region. One of these is the supernumerary mammary gland tissue more or less perfect or rudimentary, found occasionally, and the other structure constitutes the large convoluted, tubular sweat glands, which are constantly present.

The most complete recent exposition of the subject of supernumerary mammary glands in the human subject is that by W. R. Williams in the Journal of Anatomy and Physiology, Vol. xxv, 1891. It is shown that there may be one, two, or three of these above the normal gland on a line diverging outward to the axilla-two on the border of the pectoral muscle, and one in the base of the axilla. The latter is the least frequent. Most frequently these supernumeraries are rudimentary masses lying wholly beneath the skin and neither visible nor palpable, resembling in gross appearance ordinary subcutaneous connective tissue, but microscopically they show some of the normal elements of the gland. These subcutaneous "mammary lumps," as they have been called, may be wholly detached from each other and from the normal mam. mary gland, or they may be joined to the latter by an isthmus of the same kind of rudimentary tissue. In 132 consecutive cases of carcinoma in the mam. mary region in females Williams found that 13 of them had their origin in supernumerary structures quite outside the normal glands. None of these, however, were located in the axilla proper.

The sweat glands of the human axilla have been specially described by such anatomists as Sappey, Koelliker and others, but it remained for C. H. Creighton to demonstrate their peculiarities and em. phasize their significance from a pathologic stand. point. In the Royal Medico-Chirurgical Society transactions of 1882, Vol. lxv, page 53, he reports three cases of axillary carcinoma in dogs, originating in the large axillary sweat glands. Spencer Wells, in Wood's Monographs, Vol. iii, No. 1, 1889, reports a case of axillary carcinoma with the microscopic findings which seem to demonstrate that it originated in the large, tubular sweat glands in the manner described by Creighton. They are regarded as a kind of rudimentary organ of cutaneous secretion in the human when compared with similar structures in the lower animals, and, as Wells observes, are subject to all the risks of such survivals or rudiments. These glands, while also found in the groin and in the areola of the breast as well, are looked upon as midway in structure, with their large acini and amount of secreting surface, between the ordinary sweat glands and the mammary gland. They have their distinguishing histologic features by which they can be identified with ease. They are situated under the skin and not in the skin like other sweat glands and their ducts open into the hair follicles. They are large enough to be detected as firm, round bodies in the subcutaneous tissues in a dissection. It is quite probable that a more careful study of specimens of primary axillary tumors would show these glandular structures to be the point of departure for the morbid process in the majority of cases, rather than the supposed axillary supernumerary mass of mammary tissue. A study of the clinical histories also of those cases which have been put on record is suggestive in this same direction. The two following cases have served as a basis for this brief paper on this subject:

Case 1.-Miss DeC., aged 50 years, came into my hospital service in June, 1898. The right axilla was filled with a hard mass which was already causing edema of the arm by pressure. 